\title{
Urgences
}

\section{De A à Z, Collectif}

\section{Collectif}

Numéro 13, mars 1986

\section{Éclats d'atelier}

URI : https://id.erudit.org/iderudit/025241ar

DOI : https://doi.org/10.7202/025241ar

Aller au sommaire du numéro

\section{Éditeur(s)}

Urgences

\section{ISSN}

0226-9554 (imprimé)

1927-3924 (numérique)

Découvrir la revue

\section{Citer ce document}

Collectif (1986). De A à Z, Collectif. Urgences, (13), 89-104.

https://doi.org/10.7202/025241ar d'utilisation que vous pouvez consulter en ligne.

https://apropos.erudit.org/fr/usagers/politique-dutilisation/ 


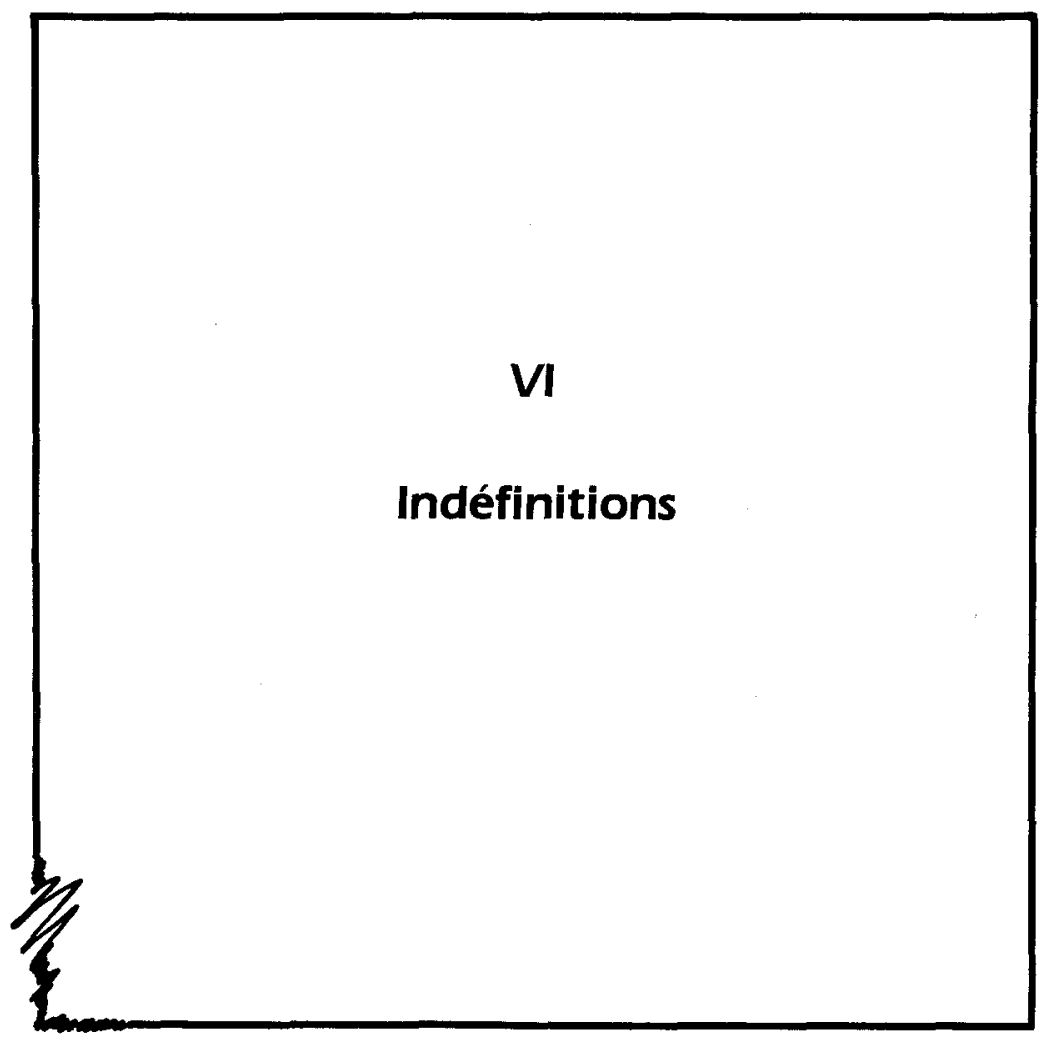




\section{Postulats: indéfinitions}

Monter, collectivement, un glossaire - ici, environ cent vingt-cinq entrées - où les mots les plus courants sont redéfinis, c'est-à-dire indéfinis selon quelques-unes des connivences de leur signifiant avec leur signifié, mais surtout avec le signifiant et le signifié d'autres mots.

À chaque fois: le mot, la catégorie, la définition, l'exemple, au moins.
$A B /$ Anne Bernier
$\mathrm{DH} /$ Danielle Hawey
$C B /$ Céline Bouchard
BL Brigitte Leblanc
LB/ Louise Beauchamp
MU Monique Lapierre
$\mathrm{MC} /$ Marie-Rose Comeau
$\mathrm{CM} /$ Chantal Morin
LD/ Lévis Dumais
$\mathrm{MM} /$ Manuel Marienval
MD/ Marie Desrosiers
$\mathrm{CN} /$ Chantal Nadeau
$\mathrm{RD} /$ Roberto Dionne
NP/ Nancy Philibert
AG/ André Gervais
MR/ Maurice Richard
DG/ Danielle Grenier
CS/ Claudette Sirois
LG/ Lise Gagnon
MG/ Mireille Goulet
DS/ Danielle Saint-Cyr
VG/ Vianney Gallant
ST/ Sonia Talbot

Glossaire - voir Michel Leiris: Glossaire jy serre mes gloses (repris dans Mots sans mémoire, Paris, Gallimard, 1969, p. 71 et ss) et son supplément: Langage tangage, Paris, Gallimard, 1985, p. 7 et ss.; voir aussi Gérard Genette: Mimologiques, coll. "Poétique", Paris, Seuil, 1976, p. 362 et ss. 


\section{A}

Adage. n.m. Vieux sage populaire qui professe, en les formulant lapidairement, des règles de conduite et de morale, et des jugements divers. "Maxime est là, comme une douce hallucination: tout ce que ce vieil adage nous a raconté me revient lentement en mémoire" (Marcel Proust). (AG)

Amour. n.m. Précipice rarement rencontré au coin des rues. "Il est tombé en amour". (ST)

Animatrice. n.f. Bête de scène. "Ardente, féline, l'animatrice tenait "son" public". (DS, AG)

Apatride. n. Individu qui pâtit, patraque, dans l'apathie patibulaire du pattern de l'apartheid. "L'apatride, entre le patron qui se graisse la patte et la patrouille qui l'appâte, est comme un pâtre sans pâture". (DH)

Arthrite. n.f. Vieil art. "Les gens de l'âge d'or exposent leur arthrite". (ST)

Atome. n. Domicile; (fam.) chez-soi, vie intime. "Qui n'aime pas se retrouver atome?" (LB)

\section{B}

Bague. n.f. Lien de fer que l'on met au doigt des prisonniers. "Il lui a passé la bague au doigt". (RD)

Bancaire. n.m. Petit banc fabriqué au Caire. “La seule chose que j'ai ramenée des pays arabes est un bancaire; il m'a probablement causé plus de problèmes là-bas qu'un faux cheik (I) m'en aurait causé ici". (CM)

Berlingot. n.m. Masse de métal précieux en forme de langue. "C'est dans les buildings de la capitale d'une Allemagne désormais irrémédiablement divisée que les berlingots (et, bien sûr, les Berlingoths) parlent la langue du pouvoir monétaire européen". (CN, AG)

Bidet. n.m. 1 Petit ventre. "Il aime prendre du bidet". 2 Succès moyen. 
" "Je me lave les mains de (et dans) ce bidet", clame le metteur en scène". Syn. Fourneau. (ML)

Bonnette. n.f. Servante de petite taille et très dévote. “Le curé baisa sa bonnette et quitta le presbytère". (DS)

Bouteille. n.f. Période de la vie marquée par une grande capacité d'émerveillement. (Pop.) "Lui, y est au boutte, eille!" (MR)

Brasier. n.m. Nom générique de la petite pièce de bois enchâssée dans le sémaphore et servant à retenir le filin de soutien des drapeaux signalisateurs d'un navire. "Le brasier céda sous la pression du vent". (NP)

Cabaret. n.m. Lieu où certains oiseaux de nuit couvent, voire même pondent leurs oeuvres. "N'est-ce pas au cabaret que, arrêtant son cabotinage, Baudelaire vola "L'âme du vin"?" (ST, AG)

Calebasse. n.f. Se dit de la partie la plus profonde de la cale d'un navire. "La calebasse était pleine de marchandises exotiques". (NP)

Charme. n.m. Arme. "Il tomba sous son charme". (MC)

Chauvin. n.m. Boisson nationale. "À chacun sa patrie: chaud, il se prend pour ledit vin". (ML, AG)

Chiendent. n.m. Caractère exécrable. “Ce gamin bien planté a du chien d'dans". (CM)

Chuchoter. v. Tomber malencontreusement par petits bonds successifs. "Dans l'escalier, un homme chuchote". (MC)

Circonférence. n.f. Allocution dont tous les énoncés, tacitement, sont à égale distance du sujet traité, et dont le commencement et la fin, textuellement, coïncident. "C'est en repensant, un peu moins circonspect, aux différents aspects de cette circonférence que je compris enfin cette notion si simple d'éternel retour" (Friedrich Nietzsche). (AG) 
Circulaire. n.f. Aire (et erre) de circulation. "Les exhibitions de ce petit cirque en plein air se font à une circulaire étourdissante". (BL, AG)

Claque. n.f. Fard. "Après quelques claques, ses joues rougissaient". (MC)

Compliment. n.m. Petit meuble à trois pattes, facile à basculer. "Elle ne releva pas le compliment". (MC)

Comportement. n.m. Manteau à compartiments qu'on porte pour se comporter envers la compagnie selon le compromis. "Son comportement est tel qu'il change d'idée comme il change de mise". (DH)

Conformité. n. Individu atteint d'une maladie incurable, analogue à une m.t.s. "Le con, fort mité, s'accomode fort mal de telle mâle aise" (L'abbé Bite). (MM)

Confort. n.m. Con et fort béatement. "Gros bras, p'tite tête: le confortl". Con et fort deux fois (voir réconfort). (ML)

Conservatoire. n.m. Conserverie d'instruments aratoires. "Au conservatoire, on apprend à agir de conserve - on dit aussi de concert: moisson et lumière - avec le choeur de champs". (DH, AG).

Conversation. n.f. Mets chinois. "Ils entamèrent une conversation à bâtons rompus". (MC)

Cosmonaute. n.m. Type d'agent soviétique réputé pour son lunatisme. Son nom lui vient de son expression habituelle: "Kosse mâ noter sur mon rapport, là?" (DH)

Coulisse. n.f. Ce qui se dit dans ses couloirs se glisse à l'oreille; attention à la licence. Tout à coup, silence. "Les cous se tendent, lisses, vers le rideau qui glisse sur le pôle coulissant. Á l'issue de l'acte, l'histoire nous dira si c'était un navet en coulis". (DH)

Crépuscule. n.m. Animal à l'arrière-train crépu. "Ravi, I'homme considérait le crépuscule". (MC) 
Criminel. n.m. Arbre au fruit rouge et amer; doit parfois subir une coupe sévère. "Ils ont guillotiné le criminel". (MC)

Crustacés. n.m. pl. Aliment cru. (Pop.) "C'pas bon manger cru, c't assez là!" (CM)

\section{D}

Décadence. n.f. Danse ancienne caractérisée par une dizaine de petits pas serrés suivis d'une chute du corps. "La Rome des cadences s'écroula, de décade en décade, en 476". (ML)

Décalage. n.m. Action de retirer marchandises et autre fret de la cale d'un navire. "Lors du décalage, le capitaine découvrit la fraude". (NP)

Décocher. v. intr. Descendre du coche. "Décrocher sans air". (ML)

Déprime. n.f. Sensation désagréable d'être mis en solde. "Sa déprime lui vaut d'être laissé sur les tablettes". (DH)

Doléance. n.f. Élancement douloureux se localisant le plus souvent dans le dos et occasionnant un inconfort suffisamment marqué pour nécessiter l'arrêt provisoire de toute activité. "Malgré ses doléances, on le contraignit à poursuivre l'escalade". (DG)

\section{E}

Écureuil. n.m. Grande queue servant à se rincer l'oeil. “Ou'il est agréable de voir un écureuil grimper". (CN)

Emballer [ $s^{\prime}$ ]. v. pron. S'offrir en cadeau avec empressement, de son plein gré. "Cette boîte m'emballe!" S'offrir, mais en conserve, pour une plus longue durée (voir remballer). (AB)

Eruciforme. n.m. Constructiviste russe. "Le plus grand des éruciformes n'a-t-il pas été celui qui a conçu ce qu'on appelle aujourd'hui, couramment, les "montagnes russes"?" (CB, ML)

Éternité. n.f. Été si terne qu'il paraît sans fin. “On nous avait promis des vacances paradisiaques, elles se révélèrent n'être qu'une éternité de plus". (DG) 
Extravagant. n.m. Perturbation des courants marins qui se produit lors des équinoxes, caractérisée par des vents violents et de la pluie. "La mer des Antilles est souvent la proie d'extravagants". (NP)

\section{$\mathbf{F}$}

Ficelle. n.f. Petit animal d'Afrique du Sud chassé essentiellement pour l'élasticité de ses fibres intestinales dont on se sert en cordonnerie. "Pendant toute la durée du safari, c'est lui qui tira les ficelles". $(A B)$

Fission. n.f. Secousses séismiologiques ayant pour conséquence l'interruption, entre les deux encéphales, de l'intersubjectivité. “La fission de l'être est une amnésie de l'inconscient ostracisé". (MM)

Flou. adj. Se dit de l'état d'âme de l'amoureux transi. "Il devient fou sans elle". (ML)

Focaliser. v.tr. Ariser la surface des focs en y prenant un ou plusieurs ris. "L'homme, du gaillard d'avant, focalisait les voiles". (NP)

Frère. n.m. Être dépressif, habitant généralement les rues principales, persécuté depuis des siècles par différents adeptes de la tempérance. "Faut-il battre le frère pendant qu'il est chaud?" (AB)

Fripon. n.m. Petit jupon au rebord frisé. "Elle est bien coquine, pourtant, cette Alice au corsage et au fripon toujours blancs comme lys". (LG, AG)

\section{$\mathbf{G}$}

Gigoter. v. tr. Cuisiner un gigot de boeuf, d'agneau ou de chevreuil. “Le cuisinier gigotait si bien que la clientèle augmentait de jour en jour". (DS)

Guérison. n.f. Établissement où se retrouve tout ce qui est nécessaire à la construction, la réparation et l'armement des navires. "En temps de guerre, il n'y a qu'une voie: de la guérison au quai d'embarquement". (NP) 


\section{H}

Hockey. $1^{\circ}$ Interj. faisant écho et servant de support à notre affirmation nationale. "Okél Okéll Okéll!" Anton. Nietl 2 (Pop.) "Ah qu'est (var. Hoquet'es) belle!". $3^{\circ}$ N.m. Lieu où I'on pêche. "On va pêcher, oké, sinon m'en vas m'choquer!" (VG)

Homme-sandwich. n.m. Homme pris dans une fâcheuse situation; coincé. "Depuis l'affaire scandaleuse du thon avarié, le ministre n'est ni plus ni moins qu'un homme-sandwich". (LB)

Hoquet. n.m. Petit jouet de forme plus ou moins allongée, qui fait un léger bruit quand on l'agite. Chose futile, qui amuse. "Depuis le berceau, il ne cesse de s'amuser avec son hoquet". (LB)

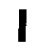

Iguane. n.m. Goût idoine. "Ici, au Mexique, notre iguane pour la fainéantise et la crêvasserie est incorrigible. À chaque fois que j'en ai l'occasion, j'aime bien le rappeler, dans une petite lettre, à Rodrigue ou Anne". (AG)

Imbu. adj. Non bu; imbuvable. "Malgré le tonneau mis en perce, il est imbu de sa personne". (AG)

Impact. n.m. Imprévisible actualité. "Dans le proverbe "Point d'impact, malgré le bonheur", que faut-il entendre: II y eut impact, malgré le bonheur (version dysphorique) ou II n'y eut point d'impact, malgré le bon heurt (version euphorique)? Ou encore, pourquoi pas, l'une et l'autre version, simultanément ou successivement, comme c'est souvent le cas dans la vie courante?" (AG)

Interdit. n.m. Ce qui est dit entre. "Il y a de l'interdit dans ses poèmes". (LD)

\section{J}

Jonque. n.f. Voilier à la quille de jonc et aux latitudes de bamboula. S'il n'y a, ici, en Occident, qu'Hamlet - et non camelote -, il ne pourrait bien y avoir, là, en Orient, que jonque aux vieux cordages et que jonction, désormais extrême, entre l'exotique flamboyance d'un soleil toujours levant et la mort toujours recom- 
mencée d'un mythe" (Mircea Eliade). (AG)

$\mathbf{K}$

Karaté. n.m. Acte manqué. "Le coup de la carotte (et du lapin sur le lit) ou le coût du carat (et du lapis-lazuli), dans l'un et l'autre cas, c'est, de toute manière, du karaté! Freud, d'ailleurs, ne l'aurait-il pas appelé, retour d'un de ses nombreux voyages, japsus fou lapsus japonais)? (AG)

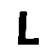

Lange. n.m. $1^{\circ}$ Volatile servant à envelopper le silence. "Un lange passa". $2^{\circ}$ Litote au miroir. "Ne fais pas la bête!" (VG)

Libertaire. adj. Qui est en faveur de la libéralisation de toutes les terres du pays. "La Commission de protection du territoire agricole est loin d'être libertaire". (LD)

Linotype. n. Type parfait du lino (ou linot), oiseau échassier longiligne tenant à la fois du lion et du Ihinocéros, et peuplant autrefois le sud de l'Éthiopie. (Malgré sa réputation de distrait, les migrations du linotype étaient particulières à cette espèce, se déplaçant par petits groupes, en ligne droite, guidés par un pilote (ou type).) Se dit aussi d'une personne étourdie. "C'est une tête de linotte type". (LD)

Litière. n.f. En Amérique du Nord, matériel le plus fréquemment utilisé pour la confection des matelas; en vogue surtout depuis 1929. "Matelas fait de litière à 100\%; sommeil absorbant, à lit et nation garantis!" (LB)

Lubrique. adj. Luxurieuse rubrique des "petits journaux" où est "dévoilée", souvent photo à l'appui, la vie privée des travailleurs. "J'écris brique et, déjà, vous avez lubrique". (AG)

\section{M}

Macadam. n.m. Attitude type du mâle. "Adam Maquennerosse, dit le mac à dames, grand imprécateur devant l'éternel et grande raquéquette". (MM, AG) 
Misogynie. n.f. Atavisme difficilement identifiable et anti-sartrien, seIon que l'être est Verbe ou complément. "Le misogyne nie l'être". Vx. Entrepreneur immobilier. "Ils les ont mises au gynécée". (DH)

Mistigri. n.m. Souverain de la Perse et mythomane célèbre qui, après la conquête de l'Egypte, voulut éliminer le dieu Râ. "Son nom, par un calembour bilingue qu'il n'est pas inutile de faire paraître ici, vient d'une réplique célèbre d'un non moins célèbre drame de l'époque élizabéthaine: "Would you play, Mister Greed, for me?"" (ML, AG)

Mitaine. n.f. Petite de la mite. "En fouillant dans mes vêtements, j'ai trouvé des mitaines". (CN)

Mort. n.m. Céréale qui sert à la fabrication de boissons alcoolisées. "On emporta le mort, pour le mettre en bière". (MC)

Motel. n.m. Maison à l'enseigne des mots. "Il n'y a pas de meilleurs endroits pour apprendre que dans un motel". (RD)

\section{$\mathbf{N}$}

Nimbus. n.m. Petit véhicule à quatre roues motrices servant au transport des nains. "Le ciel était si bas, si encombré que les nimbus avaient de la difficulté à se frayer un passage". (CS, AG)

Niveau. n.m. Espèce de nid où la vache protège son petit. "Sur le plancher, des vaches sont à leur niveau". (BL, $A G$ )

Notule. n.f. Annotation légère, très fine, à un texte, "Le texte, étymologiquement un tissu (social), présuppose l'articulation du je de l'auteur au nous de la collectivité, qu'elle soit mince frange élitiste ou large pan populaire, et ne peut être pleinement saisi sans l'apport multiplié de notules" (Lucien Goldmann). (AG)

\section{0}

Ombrelle. n.f. Objet singulier de l'immolation amoureuse. "Laissemoi devenir l'ombre de ton ombrelle". (Le proverbe - un alexandrin, l'a-t-on remarqué - est récent et renvoie, par calembour (ton ombrelle /ton nom, Brel), aux vers de la chanson bien connue 
("Ne me quitte pas"); il désigne, délicatement, cet état de perte et d'anéantissement dans lequel peut se trouver l'individu qui place tout ce qu'il est et tout ce qu'il a en l'autre .) (MD, AG)

Ordure. n.f. Variété moléculaire de l'or; elle peut atteindre 28 carats à l'état pur. "lls mettent leur ordure à la banque". (ST)

Orgueil. n.m. Instrument de musique moderne, à oeil électronique. "Je ne puis plus jouer, mon orgueil en a pris un vilain coup". (CS)

Ovaire. n.m. Récipient. "Il fait cul sec à chaque ovaire”. (BL, $A G)$

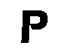

Paradis. n.m. Officines paragouvernementales où vit telle espèce rare de rat se nourrissant exclusivement de légumes. "Brian Mulroney songerait-il à quitter ce qui lui semblait être le paradis, pour aller cultiver son propre jardin?" (CS)

Patinoire. n.f. Bar réservé aux Blancs. "La patinoire est blanche de monde". (MR)

Patrouille. n.f. Formation d'un corps policier dont la stratégie consiste principalement à travailler en groupes, ce qui a donné naissance au dicton: "Quand ils y mettent la patte, ils flanquent la trouille". (DH)

Pelle. n.f. $1^{\circ}$ Relation privilégiée. "Sens-tu la pelle de mon amour?" $2^{\circ}$ Femme exagérément serviable. "La pelle du foyer". (MR)

Péninsule. n.f. Tristesse inconsolable. “Errer comme une âme en péninsule". (AG)

Pétoncle. n.m. Époux de la pétante. "Les yeux pétillant de plaisir, ma pétante et mon pétoncle, à la première occasion de sortir de leur pétrain-train routinier, sont partis en voyage sur la côte." (BL, $A G)$

Plagiat. n.m. Arrivage, parmi les embruns et les coquillages, des vols littéraires. "Qui dit plagiat dit plage, hier en tout cas". (MG, AG)

Polissonner, v. intr. Sans avoir affaire aux forces de l'ordre, sonner. 
“Très vite, il apprit à polissonner aux portes". (DG)

Politique. adj. Se dit des nombreux parasites de la peau des chiens. "Cette bête politique suce le sang du pouvoir, de notre pouvoir" (Louis Laberge). (LG, AG)

Poterie. n.f. Pot dans lequel on sert du riz. "Il y avait sur la table une poterie garnie au poulet". (BL)

Pouilles. n. f. pl. Chanson profane d'un genre injurieux, qui s'utilise souvent entre adultes. "Ouille, ouille, je me suis fait chanter pouilles par mon amoureux!" (CS)

Principe. n.m. Cousin éloigné de l'âne, il fut rapporté de France par les premiers explorateurs; bête de somme à l'origine, il fut par la suite utilisé par différentes congrégations à des fins plus récréatives. “Dès son réveil, Mère Marie de I'Incarnation aimait bien être à cheval sur les principes". $(A B)$

Prodigieusement. adv. D'une manière professionnelle et disgracieuse. "Les parents écoutent prodigieusement leurs enfants". (CB)

Professer. v. tr. Gagner sa vie en tapant sur les autres. "Les Frères du Sacré-Coeur étaient passés maîtres dans l'art de professer". (LD)

\section{$\mathbf{Q}$}

Quadrette. n.f. Équipe de quatre personnes servant à parer à toute éventualité quant à la direction d'un navire. "Pour donner un coup à tribord par un vent debout, notre quadrette n'a pas sa pareille". (NP, AG)

Quenouille. n.f. Sorte de pâtes alimentaires en forme de fuseau, cultivées chez les surréalistes et dont la découverte revient à Raymond Oueneau. "Le chiendent pousse parmi les quenouilles". (CS) 


\section{$\mathbf{R}$}

Récuser (se). v. pron. S'user à trop récurer. "Autrefois, en tant que femme à part entière, la ménagère se récusait". (ML, AG)

Religieuse. n.f. Femme qui est pure, manque d'expérience et passe un temps d'épreuve avant de prononcer des voeux. "Devenu vieux. le père, sous les yeux envieux de sa Christine, se tape une religieuse dans la sacristie". (MM, AG)

Rencart. n.m. Espèce de placard où l'on range les petites salopes. "Exaspéré, il mit sa salopette au rencart". (ST)

Rideau. n.m. Plante de la famille des graminées, cultivée depuis la Deuxième Guerre en Allemagne; son grain, riche en fer, est à la base de la locution "rideau de fer". "Le rideau a éloigné le mythe des céréales douces". (MR)

Rococo. interj. Expression tribale, demande emphatique signifiant un rappel pour un deuxième tour de chant du coq. "À tut-tête, ils s'époumonnaient: "Rococo, rococol", et le con de répondre: "Coco ri, coco ri, coco ri co!" (MM)

Romanesque. $n$.f. Fresque romaine restaurée sur de petits tableaux de pierre $(81 / 2 \times 11)$. "La plus prestigieuse collection de romanesques appartient au Centre national de prêt d'oeuvres d'art d'Italie". (CB)

Rumeur. n.f. Gratte-ciel. "Une rumeur s'élève sur la place". (MC)

\section{$\mathbf{S}$}

Salaire. n.m. Lieu malpropre où les rapaces achètent la force de travail. "C'est tout ce que j'avais comme salaire". (ST)

Salivaire. n.f. Salve salace d'herbages verbeux pour vertige livresque. "Une grande salivaire pour, sij'ose dire, s'humecter la voix". (AG)

Sauternes. n.m. Épreuve du délicathlon. "Il était pâle, il était blanc, il en était au sauternes, au coup sans éclat. N'allait-il pas décider que..." (MC, AG) 
Schizophrène. n.m. Arbre au tronc fendu et dont les branches, touffues et repliées sur ce tronc, font en sorte qu'on peut difficilement entrer en contact avec la rugueuse réalité de celui-ci. "Le schizophrène pleureur, en terrain frais et substantiel, croît communément seul" (Frère Marie-Victorin). (AG)

Solde. n.f. Ode, dite à rabais, composée durant la Deuxième Guerre par des soldats, poètes pour la circonstance. "Cette veuve de guerre ne se lasse pas d'écouter son amour mis en solde". (MDI

Sourire. n.m. $1^{\circ}$ Rongeur. Animal particulièrement sociable qui reste toujours la bouche ouverte. $2^{\circ}$ Ivresse se moquant de la malpropreté. "Le sourire est le propre de I'homme". (VG)

Spot. Abréviation d'une étrange locution qui signifie à la fois "ce n'est pas de..." et "vérifie..." (Pop.) "Spot tes afféres". (LD)

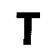

Taie. v. Leitmotiv terre à terre sur lequel se sont reposés certains hommes politiques québécois, Duplessis en tête. "Toé, tais-toél" Anton. Athée. (VG)

Terroriser. v. tr. Exprimer une passion artistique vis-à-vis de la matière dont nous sommes issus. "Le sculpteur fougueux terrorise son modèle". (ML, MM)

\section{$\mathbf{U}$}

Urbain. n.m. Plongée primordiale. "N'est-ce pas à Rome, métropole païenne, que le nommé Ben-Hur, Juif, prit son premier urbain de foule?" (AG)

Usurpation. n.f. Maladie héréditaire et incurable, symptôme d'immuno-déficience acquise. "L'usurpation est propre aux anémiques chroniques; il en résulte une usure des goûts, des passions et des sens nécessaires à la jouissance". (MM)

Utile. n.m. Ustensile dont le sens, voire l'essence même ont été bouffés. "Pour ce faire, n'aura-t-il pas fallu "joindre l'utile à l'ogre Réal" et, ainsi, sur cette étrange locution, bâtir l'incroyable conte 
que I'on sait?" (Luc Lacourcière). (AG)

\section{$\mathbf{V}$}

Velours. n.m. Tunique interne de l'oeil, qui vêt le regard de douceur, de légèreté. "Tu roules, le vélo a ses roues, l'ourse ses oursins, la louve ses loups de mer, le voleur sa ruse... Tout fait patte de velours... Alors, l'oeil, tu l'ouvres?" (Marcel Pagnol). (AG)

Venir. v. intr. Se produire entre les aines. “Tandis qu'il répétait inlassablement de venir, elle ne comprenait pas ce qu'il disait, étant là sous son corps et ne sachant pas où il voulait qu'elle aille". (BL, AG)

Vermine. n.f. Rongeur infernal, gouverné par les dieux (par Minerve plus particulièrement), qui s'attaque à l'esprit. “La vermine mine la verve". (MM)

Vibraphone. n.m. Instrument suggestif qui accompagne le saxophone. "De peur que le sexe à piles des convives "disjoncte" à cause du vibraphone, éviter le surchauffement. Le danger pour la santé et le plaisir croît avec l'usage". (MM)

\section{$\mathbf{W}$}

Wagon-tombereau. n.m. Nouveau design de cercueil à roulettes; voiture de sport gardant les lignes aérodynamiques du corbillard. "Le wagon-tombereau apparaît aujourd'hui le moyen le plus expéditif pour enrayer cette formidable croissance démographique". (MM)

Wallon. n.m. Ornithologiste. "Le wallon braquait sa lunette d'approche sur les flamants". (MC)

Wigwam. n.m. (Situation d'angoisse précédant le) "Oui, madame". "Quand, sur un coup de tête, on quitte la tribu, sujet difficile et verbe haut, il est particulièrement difficile de revenir au wigwam". (AG) 


\section{$\mathbf{X}$}

Xénon. n.m. Négation la plus lourde qui pèse sur l'essence même de l'existence; voir néant. “Ce xénon est une contingence absurde, le ciel va nous tomber sur la tête" (Assurancetourisque). (MM)

Xérès. n.m. Instrument de musique. "Le chef Charles-Henri Bertoit n'oublia pas la touche de xérès". (MC)

Xylophone. n.m. Perte de la patrie et de la voix. "Les bas guets-apens ont déjà frappé sur les lames de fond du changement social quand les prisonniers politiques jouent du xylophone". (AG)

\section{$\mathbf{Y}$}

Yeux (D'). n.m.pl. Lieu dit à notre ressemblance; miroir créé par l'ennui. "N'éteinds pas, je n'ai D'yeux que pour toi". (Pop.) "Se passer un D'yeux seul me voit". Intensif propre aux aveugles: "Bonyeux!" (VG)

Yo-yo. n.m. Traumatisme d'origine espagnole caractérisé par des bégaiements narcissiques à effet de boomerang. Anton. "Je est un autre"; tutu (ces deux antonymes ont comme propriété commune d'être sujets à des hauts et des bas). Plur. Nounou. "L'homme est un nounou pour l'homme" (stade régressif). (VG)

\section{$\mathbf{Z}$}

Zéro. n.m. Poids lourd. "Il suffit d'ajouter un zéro". (MC)

Zigzag. n.m. Couple, union plutôt bizarre. "Tout entre en ligne de compte, quand on s'aime; mais quand on sème la zizanie, quelque chose comme une ligne de vie(s) brisée(s) - en un mot: un zigzag - se met en place" (Marcel-Marie Desmarais, ptre). (AG) 